\title{
Philosophiques
}

\section{Indication formelle et déformalisation}

\section{Laurent Villevieille}

Volume 44, numéro 2, automne 2017

URI : https://id.erudit.org/iderudit/1042339ar

DOI : https://doi.org/10.7202/1042339ar

Aller au sommaire du numéro

Éditeur(s)

Société de philosophie du Québec

ISSN

0316-2923 (imprimé)

1492-1391 (numérique)

Découvrir la revue

Citer ce document

Villevieille, L. (2017). Indication formelle et déformalisation. Philosophiques, 44(2), 343-347. https://doi.org/10.7202/1042339ar d'utilisation que vous pouvez consulter en ligne.

https://apropos.erudit.org/fr/usagers/politique-dutilisation/ 


\title{
Indication formelle et déformalisation
}

\author{
LAURENT VILLEVIEILLE
}

Archives Husserl de Paris, CNRS/ENS

C'est à la fin des années dix et au début des années vingt que le jeune Heidegger forge le concept, méthodologiquement décisif, d'indication formelle. Le premier commentateur de Heidegger à en révéler l'importance fut Theodor Kisiel qui, en 1993, dans son ouvrage devenu classique The Genesis of Heidegger's Being \& Time, restitua le contenu du cours du semestre d'hiver 1920-1921 Phänomenologie des religiösen Lebens ${ }^{1}$, lequel ne devait paraître que deux ans plus tard ${ }^{2}$. Dans ce cours, en effet, Heidegger expose les tenants et les aboutissants méthodologiques de ce qu'il nomme formale Anzeige, et qu'il situe dans un débat avec la phénoménologie de Husserl. Ce débat est, du reste, très technique. Il repose sur une discussion des procédés husserliens de généralisation et de formalisation - la généralisation consistant à inscrire un objet dans la hiérarchie des espèces et des gens qui le subsument, la formalisation supposant au contraire de faire abstraction du contenu de l'objet (donc aussi de sa hiérarchie spécifico-générique), afin de ne retenir que la forme logique qui structure son objectivité. Dans la perspective de Husserl, cette forme rend compte du mode selon lequel l'objet apparaît à la conscience. En tant qu'expression du mode de conscience, elle est ainsi le lieu même de l'analyse phénoménologique, du moins telle que les Recherches logiques la définissent. Or c'est elle - la forme - que Heidegger estime nécessaire d'assortir d'un dispositif méthodologique bien particulier: le dispositif indicatif. Si la forme ne renvoie pas indicativement à la chose dont elle est la forme, elle encourt en effet le risque d'être elle-même tenue pour une chose, et ainsi de faire écran au phénomène qu'elle était initialement censée éclairer.

Ce débat avec Husserl, Theodor Kisiel se bornait à en restituer les termes. Mais pourquoi un tel débat? Quelle en est la portée? Le commentateur américain n'apportait pas de réponse claire à la question du sens et des enjeux de l'indication formelle.

C'est à cette importante question que répondent plusieurs sections de L'inquiétude de la pensée - en particulier la section intitulée "Anticipation et indication formelle» (p. 203 sq.), où Sophie-Jan Arrien, en s'appuyant en outre sur les Remarques sur Jaspers de 1919-1921, montre dans quelle

1. T. Kisiel, The Genesis of Heidegger's Being \& Time, Berkeley and Los Angeles, University of California Press, 1993, p. 164-171.

2. Phänomenologie des reliösen Lebens, Gesamtausgabe, Band 60 (désormais noté Ga 60), Francfort-sur-le-Main, Vittorio Klostermann, 1995. Le passage qui traite thématiquement de l'indication formelle, et que restitue T. Kisiel, se situe entre les pages 55 et 65 . 
mesure l'indication formelle constitue une tentative de repenser le statut des concepts philosophiques. Heidegger attribue en effet à ces concepts le statut de formes - et en ce sens, philosopher, c'est d'abord retenir la forme des phénomènes. L'indication formelle consiste dès lors à renvoyer les formes aux phénomènes dont elles proviennent, et auxquelles d'autres phénomènes pourraient, notamment en raison du poids de la tradition qui pèsent sur leur élaboration conceptuelle, mais dont la destruction a vocation à les délester, venir se substituer. Aussi Sophie-Jan Arrien peut-elle conclure (p. 210):

Tous les concepts entre lesquels louvoie le jeune Heidegger — la vie, le vécu, le Je, le soi - présentent ainsi un caractère formellement indicatif que doit libérer le travail de destruction. [...] C'est pourquoi le mérite de l'indication formelle, dans une investigation de l'origine, est sa capacité à ne pas imposer et déterminer a priori une perspective unilatérale à la recherche philosophique.

Reste alors une importante question - et c'est sur elle que nous souhaiterions faire porter ici le débat: comment s'opère le renvoi de la forme à ce dont elle est la forme? Autrement dit, comment les concepts philosophiques parviennent-ils à atteindre les phénomènes qu'ils indiquent ?

Theodor Kisiel avait bien noté, en son temps, un lien entre le concept d'indication formelle et les analyses des épîtres pauliniennes qui, dans le cours du semestre d'hiver 1920-1921, succédaient à cet exposé méthodologique. On sait en effet qu'au milieu de ces analyses extrêmement techniques, des étudiants étaient allés se plaindre auprès du Doyen de la Faculté de n'entendre parler que de méthode phénoménologique dans un cours qui était pourtant censé porter sur la philosophie de la religion. Voici en quels termes T. Kisiel commente cet épisode:

En conséquence, Heidegger coupa court abruptement — et avec irritation - à ce traitement de la formalité phénoménologique et consacra le reste du cours à la description des phénomènes religieux concrets. [...] Mais Heidegger, en pédagogue averti, se dédommagea de ses pertes en illustrant visuellement le pouvoir schématisant de son approche formelle et en mettant à nu les structures sous-jacentes qui sont opérantes dans la kotví grecque de la première épître de Saint Paul aux Thessaloniciens et, le semestre suivant, dans la rhétorique latine du livre $\mathrm{X}$ des Confessiones d'Augustin ${ }^{3}$.

Le commentateur américain fait ici allusion aux deux schémas que Heidegger avait dessinés au tableau, et qui se trouvent restitués par l'éditeur aux pages 96 et 273 du volume du cours. En "pédagogue averti », Heidegger aurait «illustré visuellement le pouvoir schématisant de son approche formelle».

Sophie-Jan Arrien a montré que le lien entre la partie méthodologique du cours et sa partie explicative était bien plus intime que ne le laissait

3. T. Kisiel, "L'interprétation formelle de la facticité: sa genèse et sa transformation ", in J.-F. Courtine (dir.), Heidegger 1919-1929. De l'herméneutique de la facticité à la métaphysique du Dasein, Paris, Vrin, 1996, p. 211. 
entendre Theodor Kisiel. Ce n'est pas dans de simples schémas que l'indication formelle continue d'être opérante: c'est dans le tissu même de l'explication que Heidegger consacre aux épitres de Paul. D'où le titre d'une section que L'inquiétude de la pensée y consacre: «Foi et indication formelle», titre qui reprend d'ailleurs celui de la contribution de Sophie-Jan Arrien à un collectif publié quelques années plus tôt ${ }^{4}$. Heidegger ne trouve pas en effet dans la foi paulinienne une simple occasion d'appliquer son dispositif méthodologique d'indication formelle: son analyse de la foi paulinienne entretient un lien structurel avec ce qui, en elle, devient plus qu'un simple dispositif. Mais quelle est la nature de ce lien?

À la lecture des pages que L'inquiétude de la pensée y consacre, il semble que ce rapport soit d'ordre mimétique. Sophie-Jan Arrien décèle en effet dans la foi paulinienne la même structure que dans l'indication formelle: l'une et l'autre impliquent en effet la suspension de toute détermination et l'attente d'un événement à venir (la parousie chez Paul, la présence du phénomène chez Heidegger). Ainsi peut-on lire par exemple page 278:

Dans le cadre de la situation propre au christianisme, la foi apparaît ainsi remplir l'ensemble des caractères de l'indication formelle en vue d'un accomplissement authentique du sens de l'existence chrétienne.

Ou encore, page 288:

Nous avons suggéré que la foi tenait, dans le contexte d'une phénoménologie de la vie religieuse, le rôle d'indication formelle originaire. [...] Cette indétermination [du contenu de la foi], revendiquée au nom du sens d'effectuation facticiel propre à la vie chrétienne, transforme précisément le «savoir de la foi » en savoir indicatif formel.

Cette interprétation, qui détermine la relation de l'indication formelle et de la foi comme une relation mimétique, nous paraît problématique à divers titres. Dans l'économie du cours de Heidegger, en effet, la foi demeure un concept. La question se pose, donc, de savoir comment le concept de foi peut indiquer formellement ce qu'il vise - et à vrai dire, cette question se pose pour lui comme pour n'importe quel autre concept. Assurément, la foi paulinienne constitue bien une déformalisation de ce que le concept de foi indique formellement. Mais là encore, cette remarque vaudrait pour n'importe quel phénomène qui, en tant qu'indiqué formellement, constitue, une fois atteint, la déformalisation de ce qui était visé au départ sur un mode seulement conceptuel. Précisément, tout le problème est de savoir comment le phénomène peut être atteint. Ce problème se pose avec la même acuité pour la foi paulinienne: comment, en 1921, dans un amphithéâtre de l'université de Fribourg-en-Brisgau, atteindre le monde de la foi au sein duquel

4. S.-J. Arrien, «Foi et indication formelle. Heidegger, lecteur de saint Paul», in S.-J. Arrien et S. Camilleri (dir.), Le jeune Heidegger (1909-1926), Paris, Vrin, 2011, p. 155-172. 
les épîtres de Paul furent écrites? Que la foi paulinienne comporte une dimension d'indétermination, de retenue et d'attente, cela est indéniable. Cependant, cette dimension nous paraît moins relever de l'ordre méthodologique de l'indication formelle, que de l'ordre phénoménal de la vie facticielle. D'où l'intérêt, précisément, de l'indiquer formellement. Mais dans ces conditions, la question demeure entière de savoir comment ce phénomène est susceptible d'être atteint par voie d'indication formelle.

Cette question, L'inquiétude de la pensée y apporte en partie une réponse lorsque Sophie-Jan Arrien relève une propriété essentielle du concept d'indication formelle: celle de suspendre l'inférence non critique d'un concept à une constellation d'autres concepts qui, s'ils semblent tout naturellement s'y rattacher, impliquent en réalité une dénaturation du phénomène. Ainsi, lorsque Heidegger veut indiquer formellement le sens de ce que «historique» signifie, il suspend l'inférence de «historique » à «ce qui devient dans le temps", et de cette dernière expression à "passé»" ${ }^{5}$ C'est d'une manière légèrement différente, mais néanmoins parente, qu'il procède lorsqu'il met en garde contre la tentation de comprendre le concept de foi à partir de sa compilation avec d'autres concepts pauliniens réputés décisifs (justice, chair) ${ }^{6}$. Sophie-Jan Arrien note ce point capital dans les termes suivants (p. 212-213):

La plurivocité des concepts, par sa fonction indicative formelle, ouvre la structure de sens et de signification différenciée des phénomènes pointant ainsi vers des domaines variés d'effectuation possible du sens. La différenciation et la poursuite de ces directions de signification, par le travail de destruction, impliquent une caractérisation qui leur soit appropriée tout autant qu'à la région d'objets dont elles émergent. (...) Évidemment, le fait de suivre une indication formelle ne permet pas de définir le(s) point(s) d'arrivée de la destruction. Au contraire, Heidegger laisse précisément ouvertes dans l'idée d'indication formelle toutes les possibilités de remplissement, mieux, d'accomplissement ou d'effectuation des significations.

Dès lors, comment atteindre le phénomène? Nous voudrions, en suivant une piste suggérée par le texte que nous venons de citer, soumettre ce point à la discussion. Sophie-Jan Arrien se réfère ici à une notion qu'elle relève dans le cours du semestre d'été 1920 , et que l'on retrouve sous des formes légèrement modifiées dans celui du semestre suivant: la notion de Bedeutungszusammenhang, de "cohésion de significations". Cette notion implique qu'un concept est une signification prélevée sur un contexte d'ensemble dont on l'excepte momentanément. L'arracher ainsi à son contexte demeure bien entendu indispensable lorsqu'on s'attache, par exemple, à expliquer une épître de Paul, ce qui implique toujours de relever tel mot plutôt que tel autre, de faire ressortir telle expression au sein de telle phrase 
où elle s'inscrit. Cependant, cet arrachement d'une signification à son contexte comporte un risque majeur: celui d'insérer cette signification dans le réseau d'un autre contexte qui, parce qu'il semble aller de soi, va insensiblement supplanter le contexte de signification originaire et authentique. L'indication formelle vise à conjurer ce risque: en suspendant toute inférence d'un concept à un autre, elle préserve l'inscription d'une signification dans un contexte qui lui serait étranger. Qu'est-ce, dès lors, qu'atteindre le phénomène? C'est avoir lentement et patiemment reconstitué les termes du contexte de signification dont on avait prélevé le concept - par exemple le concept de foi qui, lorsque le contexte de signification paulinien a été reconstruit, donne accès au phénomène de la foi proprement paulinienne. En ce sens, l'indication formelle est le suspens nécessaire à ce travail de reconstruction. La déformalisation, elle, est tout simplement le résultat de ce travail.

Si donc il nous fallait ultimement résumer le point que nous souhaitons soumettre à la discussion, nous pourrions poser la question suivante: si la foi tient, sur un plan phénoménal, c'est-à-dire dans la détermination de la vie facticielle, un rôle incontestablement privilégié, ne doit-elle pas, sur un plan cette fois non plus phénoménal, mais méthodologique, être mise au même niveau que n'importe quel autre phénomène, lequel exige, lorsqu'il est saisi conceptuellement, un acte de suspension de la détermination (indication formelle) et une lente reconstruction de son contexte de signification (accès au phénomène), reconstruction à laquelle se livre précisément Heidegger dans sa lecture des épîtres de Paul? Ce qui entraîne dans son sillage cette autre question, d'ordre plus général: comment comprendre la déformalisation? Celle-ci doit-elle, comme nous l'avons suggéré, être appréhendée comme reconstruction du Bedeutungszusammenhang, de la "cohésion de significations"? 